\title{
THE HELI-DEM MODEL ESTIMATION
}

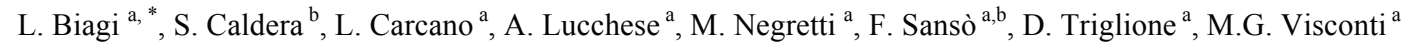 \\ a Politecnico di Milano, DICA, Geomatics Laboratory at Como Campus, Via Valleggio 11, 22100 Como (IT) \\ ${ }^{\mathrm{b}}$ GReD srl, Geomatics Research \& Development, via Morozzo della Rocca 3, 20123 Milano (IT) \\ * contact email: ludovico.biagi@polimi.it
}

Commission IV, WG IV-3

KEY WORDS: DTM, Merging, Re-gridding, Interpolation

\begin{abstract}
Global DEMs are fundamental for global applications and are necessary also at the local scale, in regions where local models are not available. Local DEMs are preferred when they are available and if are characterized by better accuracies and resolutions.

In general, two problems arise. Firstly, an interest region could be patched by several partly overlapping DEMs that present similar accuracies and spatial resolutions: they should be merged in a unified model. Moreover, even when the interest region is covered by one unified DEM, local DEMs with better accuracy could be available and should be used to locally improve it.

All these problems have been addressed within HELI-DEM project. HELI-DEM (HELvetia-Italy Digital Elevation Model) is a project that has been funded by the European Regional Development Fund (ERDF) within the Italy-Switzerland cooperation program. It started in 2010 and finished at the end of 2013. The involved institutions in the project were Fondazione Politecnico di Milano, Politecnico di Milano, Politecnico di Torino, Regione Lombardia, Regione Piemonte and Scuola Universitaria della Svizzera Italiana. One specific aim of the project was the creation and the publication of a unified Digital Elevation Model for the part of the Alps between Italy and Switzerland.

The interest area is prevalently mountainous, with heights that range from about $200 \mathrm{~m}$ to 4'600 m. Three low Resolution DTMs (20$25-50 \mathrm{~m}$ of resolution) are available that partly overlap and patch the whole project area: they are characterized by accuracies of some meters. Also High Resolution DTMs (1-5 m) are available: they have accuracies of some decimeters but cover limited areas of the project. The various models are available in different reference frames (the European ETRF89 and the Italian Roma40) and are gridded either in cartographic or geographic coordinates. Before merging them, a validation of the input data has been performed in three steps: cross validation of LR DTMs, validation of LR DTMs by HR DTMs and final check by geodetic techniques. The comparisons confirm the accuracy of HR data and the presence of few local anomalies in LR DTMs.

Considering the goal of the project and the previous results, two different DTMs have been produced. Both of them cover the whole project area (boundaries: $\lambda=7.80^{\circ}$ East and $\lambda=10.70^{\circ}$ East, $\phi=45.10^{\circ}$ North e $\phi=46.70^{\circ}$ North). They are gridded in ETRF2000 geographical coordinates and their spatial resolution is $2 \times 10^{-4}$ degrees.

The former has been obtained by interpolating and merging all the input LR DTMs on a new common grid. This DTM has been called HD-1. HD-1 presents the same local anomalies of the LR DTMs used as input for the interpolation: therefore, at least in areas where better data (HR DTMs) are available, its correction was needed. In order to avoid sharp discontinuities, corrections obtained by HR DTMs have been filtered by a numerical FFT approach before applying them. The result of this correction has been called HD-2. HD-1 and HD-2 have already been published by an open access geoservice.
\end{abstract}

\section{INTRODUCTION: THE HELI-DEM PROJECT AND THE AVAILABLE DATA}

Elevation data are fundamental in Geographical Information Systems (GIS) and one of the spatial data provided by the European Directive INSPIRE (Infrastructure for Spatial Information in Europe). They are stored in Digital Elevation Models (DEM, Li et al., 2005, El-Sheimy et al., 2005), which are the basic tools for many environmental and territorial applications. DEM refers to the generic family of elevation models: they are distinguished in Digital Surface Models (DSMs), which represent the surface (including buildings, woods, etc.) and Digital Terrain Models (DTMs) which represent the elevation of bare soil. DTMs are important in Environmental Engineering, Civil Engineering and in many specific applications, such as the design of telecommunication networks or precise agriculture.

In recent years, both in Italy and Switzerland, elevation data with different reference frames, technologies, accuracies and data models have been acquired. HELI-DEM (HELvetia-Italy Digital Elevation Model) project (Biagi et al, 2011), funded by the European Regional Development Fund (ERDF) within the Italy-Switzerland cooperation program, aims at developing a unique DTM for the alpine and subalpine area between Italy (Piedmont, Lombardy) and Switzerland (Ticino and Grigioni Cantons). The DTM will be correctly georeferenced and produced validating and integrating all the available data. The institutions involved in the project are: Fondazione Politecnico di Milano, Politecnico di Milano, Politecnico di Torino, Provincia Autonoma di Bolzano, Regione Lombardia, Regione Piemonte and SUPSI (Scuola Universitaria della Svizzera Italiana). Two other institutions, SwissTopo and the Military Geographic Institute (IGM) are involved as external partners.

The first aim of HELI-DEM project was the collection of data and metadata for the study area. In particular, this phase involved the research and the collection of local DTMs, i.e. Digital Terrain Models with medium to high resolutions, developed by local authorities (Regions, National Geodetic Authorities), with a national or regional extension. The following data have been made available to the project partners:

- initially, official DTMs of Lombardy, Piedmont and Switzerland with resolutions of 20,50 and 25 meters respectively, 
- at about the middle of the project, one more DTM with a 5 meters horizontal resolution has been acquired for Piedmont.

The DTMs are in different reference frames. The Lombardy DTM is in the Italian Roma 40 reference frame, projected in the Gauss Boaga (West zone) cartographic coordinates (R40-GB), while the other DTMs are provided in ETRF89. In particular the DTMs of Piedmont is in UTM coordinates, while the Swiss DTM is in geographic coordinates.

Since the input DTMs are not all in the same reference frame, before doing any cross-validation and merging, they have been transformed into a unique reference frame. To do that, the program GK2CNV which allows to transform a list of points or a grid from one original reference/coordinate frame to another one has been used.

GK2CNV program (Biagi et al., 2012a), developed in FORTRAN language under the GNU General Public License, allows to apply reference frames (ETRF89, ETRF2000, R40GB, ED50) transformations to spatial data, according to Italian specifications realized by the Italian Military Survey Office (IGM - Istituto Geografico Militare, Altamimi and Boucher, 2001, Donatelli et al., 2002). The transformations are based on latitude and longitude shift parameters, gridded for the entire national territory with a spatial resolution of 5 ' in latitude and of 7'30" in longitude. The vertices (more than 800) used for the calculation of the national transformation parameters among the different frames belong to the National geodetic network. In the most common case, in which the point to be transformed does not coincide with a grid node, the shift value is obtained interpolating (using a bilinear interpolation algorithm) the four nodes closest to the point. It is worth to remember that, even if the input points of a reference frame transformation are on a regular grid, the transformed points are no more on a grid although they are almost regularly distributed.

\section{CROSS-VALIDATION OF DTMS}

To cross validate the DTMs, the comparison among crossborder models with similar horizontal resolutions where they overlap has been performed. Several cross-validations between different couples of DTMs have been carried out. As case studies, in the following sections only some comparisons will be reported:

- Lombardy (res. $20 \mathrm{~m}$ ) and Switzerland (res. $25 \mathrm{~m}$ ) DTMs,

- Piedmont (res. $50 \mathrm{~m}$ ) and Switzerland (res. $25 \mathrm{~m}$ ) DTMs.

All these models have similar resolutions and accuracies: no one of them can be considered as truth and they have been compared on points that have been randomly extracted with a uniform spatial distribution within their overlaps: the horizontal density of the comparison points is about 1 point $/ 400 \mathrm{~m}^{2}$. For each comparison point two elevations have been obtained, through exact bicubic interpolation on the nodes of the couple of DTMs to be compared. For each point the elevation difference has been calculated. The analyses on the differences have been done following this schema:

- statistical analysis,

- subdivision of the differences into classes and computation of the percentage of points belonging to each class,

- spatial analysis of the distribution of differences.

2.1.1 Results and statistics of the comparison between Lombardy and Switzerland DTMs.
The CH DTM has been interpolated directly on the comparison points, which have been generated in ETRF89. Since the Lombardy DTM is in R40-GB (cartographic coordinates), the comparison points have been transformed from ETRF89 to R40-GB before the interpolation.

The sample has been considered from a statistical point of view:

$\begin{array}{llll}\text { - } \operatorname{mumber} \text { of points } & = & 256737 \\ \text { - mean: } \mu\left(\Delta \mathrm{h}_{\mathrm{L}}\right) & = & -0.1 \mathrm{~m} \\ \text { - } \operatorname{std}:: \quad \sigma\left(\Delta \mathrm{h}_{\mathrm{L}}\right) & = & 18.9 \mathrm{~m} \\ \text { - } \max : \max \left(\Delta \mathrm{h}_{\mathrm{L}}\right) & = & 352 \mathrm{~m} \\ \text { - min: } \min \left(\Delta \mathrm{h}_{\mathrm{L}}\right) & = & -257 \mathrm{~m}\end{array}$

The sample is not biased; its standard deviation exceeds the vertical nominal accuracies of the two DTMs but is comparable with them. The sample has been classified by absolute values. The result of this operation has been summarized in Table 1: more than $80 \%$ of the points presents differences smaller than 20 meters. On this regard the results are satisfactory. On the other hand, several (964) outliers $(|\Delta \mathrm{h}|>100 \mathrm{~m})$ are present.

\begin{tabular}{|l|l|c|}
\hline Class & Limits & Percentage \\
\hline 1 & $0 \mathrm{~m} \leq|\Delta \mathrm{h}|<10 \mathrm{~m}$ & $60.68 \%$ \\
\hline 2 & $10 \mathrm{~m} \leq|\Delta \mathrm{h}|<20 \mathrm{~m}$ & $23.47 \%$ \\
\hline 3 & $20 \mathrm{~m} \leq|\Delta \mathrm{h}|<50 \mathrm{~m}$ & $13.31 \%$ \\
\hline 4 & $50 \mathrm{~m} \leq|\Delta \mathrm{h}|<100 \mathrm{~m}$ & $2.17 \%$ \\
\hline 5 & $100 \mathrm{~m} \leq|\Delta \mathrm{h}|<150 \mathrm{~m}$ & $0.28 \%$ \\
\hline 6 & $|\Delta \mathrm{h}| \geq 150 \mathrm{~m}$ & $0.10 \%$ \\
\hline
\end{tabular}

Table 1. Classes of elevation differences between Switzerland and Lombardy and their percentages,

As concerns the distribution of the sample, Figure 1 shows the frequency curve of the height differences, compared to a Gaussian with the same mean and standard deviation of the sample and computed in the interval $-3 \sigma \div+3 \sigma$. Already a visual inspection shows that no good correspondence between them exists. This is confirmed by the classical chi square adapting test.

The greatest differences are concentrated in some specific areas, that do not correspond to the most mountainous areas. For example, the North-East part has higher mountains than the central one, but more outliers are present in the latter. Note that the overlaps between the two DTMs is in Lombardy, because the CH DTM covers part of the Italian region, while the viceversa does not happen. The correlations between the differences and both elevations and slopes have been computed: none of them is significant (both the values are smaller than $0.05)$. The anomalies are probably due to some problem during the realization of one of the two DTMs (i.e. errors in the digitization of the original cartography). Moreover, by other comparisons herewith not discussed in detail, Lombardy DTM contains other anomalous values in an area that experienced severe landslides in the last years (Lat $46.40^{\circ}$; Lon $10,35^{\circ}$ )

\subsubsection{Results and statistics of the comparison between Switzerland and Piedmont DTMs.}

The comparisons have been computed in the same way of the above case. The statistics of the differences are reported below:

- number of points $=824^{\prime} 057$

- mean: $\mu\left(\Delta \mathrm{h}_{\mathrm{P}}\right)=1.3 \mathrm{~m}$

- std.: $\sigma\left(\Delta \mathrm{h}_{\mathrm{P}}\right) \quad=\quad \pm 25.8 \mathrm{~m}$

- $\max : \max \left(\Delta \mathrm{h}_{\mathrm{P}}\right)=318 \mathrm{~m}$

- $\min : \min \left(\Delta \mathrm{h}_{\mathrm{P}}\right) \quad=\quad-265 \mathrm{~m}$ 


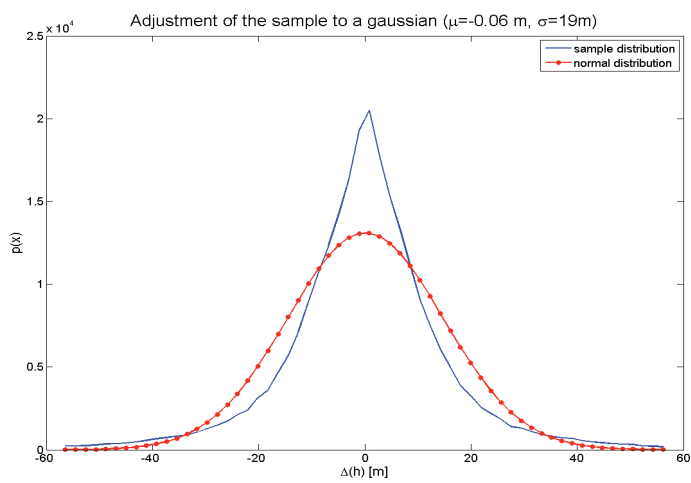

Figure 1. Histogram of the height differences (SwitzerlandLombardy DTMs) compared with Gaussian distribution with the same standard deviation.

The mean of the differences is of about 1.3 meter. This value is not significant if compared to the nominal accuracy of the datasets: however, the same bias is confirmed by Piedmont and Lombardy comparison, here not deeply discussed for space reasons. The standard deviation of the differences significantly exceeds the nominal accuracies of the two DTMs under consideration. As in the Lombardy case, the sample distribution does not fit a Gaussian distribution with the same mean and standard deviation. After the computation of the elevation differences $\Delta \mathrm{h}_{\mathrm{P}}$, the sample has been divided into the six classes used already for Lombardy (Table 2). In this case the differences smaller than 20 meters are less than $70 \%$ and more outliers are present (4'637). On the contrary, there aren't areas characterized by particular anomalies and the $\Delta \mathrm{h}_{\mathrm{P}}$ are homogeneously distributed in the area: also in this case, the correlations between the differences and heights and slopes are not significant (both the values are smaller than 0.05).

\begin{tabular}{|l|l|c|}
\hline Class & Limits & Percentage \\
\hline 1 & $0 \mathrm{~m} \leq|\Delta \mathrm{h}|<10 \mathrm{~m}$ & $39.12 \%$ \\
\hline 2 & $10 \mathrm{~m} \leq|\Delta \mathrm{h}|<20 \mathrm{~m}$ & $27.11 \%$ \\
\hline 3 & $20 \mathrm{~m} \leq|\Delta \mathrm{h}|<50 \mathrm{~m}$ & $28.10 \%$ \\
\hline 4 & $50 \mathrm{~m} \leq|\Delta \mathrm{h}|<100 \mathrm{~m}$ & $5.11 \%$ \\
\hline 5 & $100 \mathrm{~m} \leq|\Delta \mathrm{h}|<150 \mathrm{~m}$ & $0.48 \%$ \\
\hline 6 & $|\Delta \mathrm{h}| \geq 150 \mathrm{~m}$ & $0.08 \%$ \\
\hline
\end{tabular}

Table 2. Classes of elevation differences between Switzerland and Piedmont and their percentages.

\section{THE UNIFIED DTM: PREPROCESSING AND FINAL COMPUTATION}

The main final product of HELI-DEM is a unified DTM obtained through the merging of the low/medium/high resolution data. In particular the output DTM covers the ETRF2000 rectangle comprised by the following boundaries: $\lambda=7.80^{\circ}$ East and $\lambda=10.70^{\circ}$ East, $\phi=45.10^{\circ}$ North and $\phi=46.70^{\circ}$ North. The DTM is gridded in geographical coordinates with a spatial resolution of $2 \times 10^{-4}$ degrees, that means about $15 \mathrm{~m}$ in longitude and $22 \mathrm{~m}$ in latitude.

It has been obtained by re-gridding all the input low resolution DTMs that have been previously transformed in ETRF2000. The interpolated DTM (HD-1) has been corrected by high resolution DTMs where they are available to produce a second DTM, HD-2. The elaboration of the data can be synthetically schematized as the sequence of three main operations:

1. pre-elaboration, to prepare the input data,
2. elaboration (re-gridding and interpolation) of HD-1,

3. post-elaboration and correction of HD-1 for lake areas,

4. correction of possible anomalies and outliers in HD-1 by more accurate data where they are available: production of HD2.

During the pre-elaboration a first validation has been performed to verify if some duplicate points (points with coincident horizontal coordinates) exist in the datasets: this happens typically for Piedmont DTM at $5 \mathrm{~m}$ of horizontal resolution, that is stored in several files with overlaps at the borders. If this occurs, the duplicate points have to be averaged or removed. The set of duplicate points is composed in most cases by two points, but in some cases they can be also three or four. The implemented approach is to find for each group of $\mathrm{N}$ coincident points the maximum and minimum elevation values and to compute their difference; if the difference exceeds 5 meters, the $\mathrm{N}$ points are rejected and deleted from the dataset, otherwise the group is substituted by a single point which has the same horizontal coordinates of the $\mathrm{N}$ points and the mean of their elevations. Table 3 summarizes the statistics relevant to the coincident points.

\begin{tabular}{|l|l|}
\hline Number of coincident points groups & 8'839'874 $^{\prime} 805 \mathrm{~m}$ \\
\hline Mean & 0.05 \\
\hline Std & $0.39 \mathrm{~m}$ \\
\hline Maximum & $34.61 \mathrm{~m}$ \\
\hline Minimum & $0.00 \mathrm{~m}$ \\
\hline
\end{tabular}

Table 3. Statistics of the differences between the maximum and minimum elevations of the groups of coincident points belonging to Piedmont DTM with horizontal resolution $5 \mathrm{~m}$.

The three DTMs, converted into the output reference frame, have been independently interpolated on the output final grid by using local bicubic surfaces as discussed in Biagi and Carcano, (in press). In bicubic interpolation, sixteen parameters have to be estimated: therefore, to build the iso-determined interpolation system a minimum of sixteen input observations is required. A problem of ill conditioning of the system can arise when the observations have not optimal spatial configuration around the prediction node. In our dataset this has occurred quite often and we have chosen to solve by increasing the number of observations.

In a conservative choice, the bicubic interpolation is always performed using a minimum of 32 observations: therefore, Least Squares (Koch K. R, 1987) are adopted to estimate the unknown parameters. The 32 nearest points are searched inside a window of $200 \mathrm{~m} \times 200 \mathrm{~m}$ around the predicted node: therefore about 100 points for Lombardy and Switzerland and 1'600 points for Piedmont should be available. To verify if the system is stable, the normal matrix $(\mathrm{N})$ of the LS system is computed and its condition number is compared with a threshold equal to $10^{5}$. If the condition number exceeds the threshold, the input observations are iteratively incremented of 32 units, until the threshold is satisfied. Therefore, the bicubic parameters are estimated and the elevation is computed in the prediction node.

In few cases, the condition number of $\mathrm{N}$ still exceeds the threshold even when all the points of the search window have been added. In this case, a different approach is used: the number of observation is reset to 32 and a bilinear surface is adopted: this is like a drastic reduction of parameters that could 
be obtained by applying a SVD approach to reduce an ill conditioned system. The statistics of the three interpolated DTMs are summarized in Table 4.

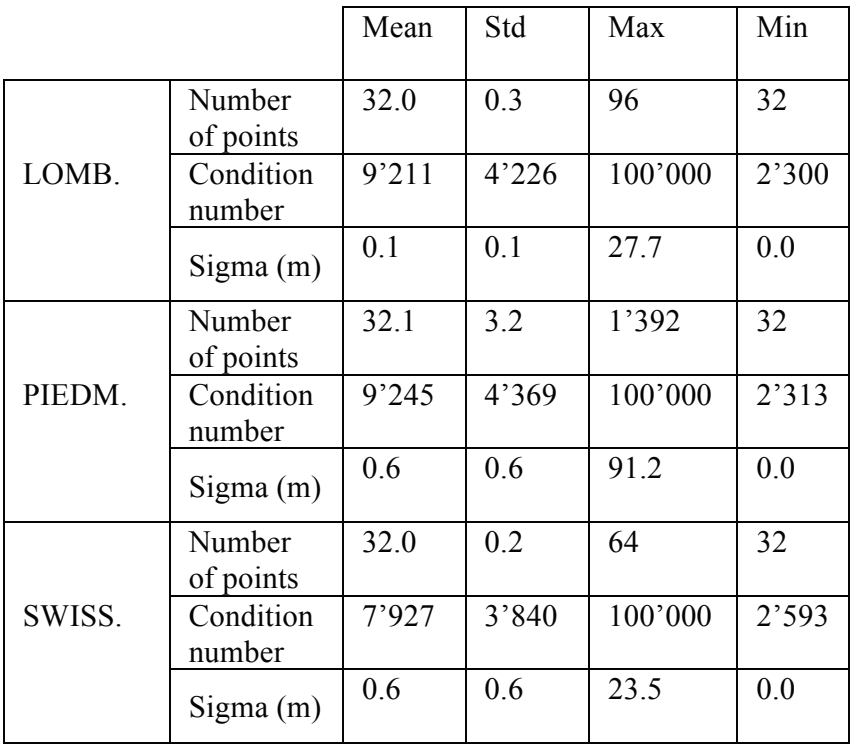

Table 4. Statistics of the results of the individual interpolation of the three low/medium resolution input DTMs on the output HD-1 grid, before the average results operation (total of 22'822'816, 33'746'321, 35'699'169 real valued nodes respectively for Lombardy, Piedmont and Switzerland datasets). Number of points: number of points used to interpolate the elevation of each node. Condition number: condition number of the final system. Sigma: final standard deviation of the interpolated heights by Least squares system.

At the borders of the regions an erosion has been performed. In fact, when interpolating input data to one output node, the observations are searched inside a square window. Therefore the interpolation is performed also for the nodes that lie near but outside the input DTM. In these cases, the interpolation could present a sufficient conditioning but is clearly not reliable. The erosion has been performed deleting, for each interpolated DTM, a strip with a width of five cells at the border. At this point the unique final unified DTM has been obtained. To do that, the three interpolated DTMs have been merged into a unique model by averaging them in their overlaps.

In total about 72 millions nodes have been interpolated and are real valued: the other nodes of the rectangular output grid $(8000$ $\mathrm{x} 14500$ nodes, for a total of $116 \times 10^{6}$ nodes) are outside the project area and assume no data value.

Points belonging to lakes have required a dedicated post elaboration. In some DTMs they are stored as a constant reference value, while in others the value is variable in space (of the magnitude of few centimeters). In addition the conventional elevation attributed to lakes can be different in the different DTMs. During the creation of HD-1 a reference constant value has been set for nodes belonging to the surface of each lake: this constant value has been computed after the interpolation of the coastlines as the minimum elevation among all the coastline points.

The interpolation of elevations near lakes has proved to be less reliable, because points belonging to lakes introduce false elevations in the input dataset and therefore in the interpolation estimate itself. For this reason for the major lakes (Como Lake, Maggiore Lake, Lugano Lake, Iseo Lake, Orta Lake) some corrective measures have been applied. For each lake, a buffer
100 meters wide, which extends from the border of the lake to the inland, has been created; then points belonging to the buffer have been interpolated using only the input points that fall inside it.

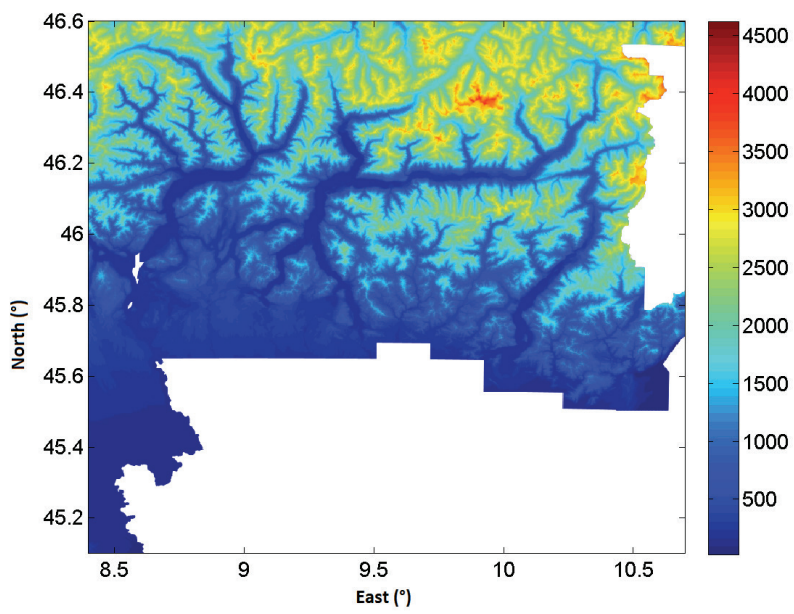

Figure 2. Unified HELI-DEM DTM (HD-1) obtained through the merging of Lombardy, Piedmont and Swiss low/medium resolution.

\section{CORRECTION WITH LIDAR HIGH RESOLUTION DTM (PST-A)}

Clearly, HD-1 still contains all the possible anomalies that were present in the input data used to interpolate it. Therefore, its local correction with more accurate data where they exist has been implemented.

In addition to the low resolution elevation data used to create HD-1, also a high resolution LiDAR DTM is available for the project. This DTM is gridded in ETRF2000 geographic coordinates, has a resolution of $10^{-5}$ degrees and a vertical accuracy better than 0.5 meters; it covers the valleys of the main river basins of Lombardy and Piedmont (Figure 3). It is produced by the Italian Department for Environment within the national remote sensing project for the Environment (Piano Straordinario di Telerilevamento Ambientale) and is named PST-A.

The problem in the correction of a DTM with a local model that has better horizontal resolution and accuracy, is to avoid jumps at the border between the zones in which the correction data are present and the zones in which they are not. It can be simply solved by properly filtering the corrections before their application. The filtering must be studied in order to produce a reasonably smooth model, which at the same time preserves as much as possible the accuracy of the correction.

The general methodology that has been applied can be shown schematically in the following way:

1. computation of the corrections (differences between high resolution and low resolution DTMs),

2. appropriate filtering of the corrections,

3. application of the filtered corrections to the model that has to be corrected.

1. Computation of the corrections. Corrections have been computed on the nodes of the HELI-DEM HD-1 DTM; to this purpose PST-A has been subsampled on HD-1 nodes. Then, their differences have been computed. In the nodes where subsampled PST-A does not exist, differences have been set to zero. In the following the model of the differences is called "corrections model". 
2. appropriate filtering of the corrections. A low pass filter allows to smooth the discontinuities in the corrections, particularly where they pass from zero to a actual value. The Butterworth filter has been used: it is given by the following formula

$$
f(i, j)=\frac{1}{1+\left[\frac{i^{2}+j^{2}}{D_{0}^{2}}\right]^{n}}=\frac{1}{1+\left[\frac{d}{D_{0}}\right]^{2 n}}
$$

$n$ and $D_{0}$ parameters are set by the user.

Actually, an iterative version has been implemented, according to (Carcano, 2014). Moreover, it has been implemented by FFT (Brigham,1974), according to the following schema:

1. computation of the model of the corrections,

2. construction of the Butterworth Filter,

3. computation of the FFTs of both corrections and filter,

4. iterated product of the two FFTs,

5. Inverse FFT (IFFT) of the product for the reconstruction of the filtered corrections.

\section{Application of the filtered corrections.}

The filtered corrections have been applied to HD-1: in the following this corrected DTM will be called HD-2.

Table 5 shows the statistics of the differences between PST-A and HD-1 / HD-2 for the whole HELI-DEM area.

\begin{tabular}{|c|c|c|c|c|}
\hline & Bias (m) & $\operatorname{Std}(\mathrm{m})$ & $\operatorname{Max}(\mathrm{m})$ & $\operatorname{Min}(\mathrm{m})$ \\
\hline $\begin{array}{c}\text { PST-A - } \\
\text { HD-1 } \\
\text { (original) }\end{array}$ & 0.3 & 6.0 & 186.8 & -174.6 \\
\hline $\begin{array}{c}\text { PST-A - } \\
\text { HD-2 } \\
\text { corrected) }\end{array}$ & 0.0 & 0.4 & 108.4 & -97.4 \\
\hline
\end{tabular}

Table 5. Statistics of the differences between PST-A LiDAR DTM and HD-1 DTM / HD-2.

This internal validation of HD-2 DTM gives very satisfactory results. For a first external validation, a set of about 1000 measures has been used. These data have been collected in Valtellina area by GNSS RTK technique (Hofmann-Wellenhof, 2008), are characterized by an accuracy surely better than $10 \mathrm{~cm}$ and have been used in a previous step of the project, to check the Lombardy DTM in zones where it has provided anomalous values (Biagi et al., 2013): this analysis is useful to evaluate if the correction actually leads to an improvement of the final product. The elevations of the GPS points (943 points in total) have been collected and merged into a unique dataset. Then HD-1, the subsampled PST-A and HD-2 have been interpolated by bicubic interpolation on the GPS points. Therefore, the differences between the three models and the GPS elevations have been computed.

\begin{tabular}{|c|c|c|c|c|}
\hline & Bias $(\mathrm{m})$ & Std $(\mathrm{m})$ & Max $(\mathrm{m})$ & Min $(\mathrm{m})$ \\
\hline HD-1 - RTK & 3.4 & 5.5 & 24.2 & 0.0 \\
\hline PST-A - RTK & -0.3 & 1.0 & 7.2 & 0.0 \\
\hline HD-2 - RTK & -0.4 & 1.7 & 8.8 & 0.0 \\
\hline
\end{tabular}

Table 6: Statistics of the modulus of the differences between HD-1, PST-A and HD-2 DTMs and RTK.

The statistics prove the reliability of the applied correction: the differences between HD-2 and RTK are significantly better than those of HD-1 and are comparable with those of PST-A.

\section{CONCLUSIONS}

The presented research is in the framework of the Interreg ItalySwitzerland HELI-DEM (Helvetia-Italy Digital Elevation Model) project. The project aim was the merging of the available elevation information into one DTM relevant to a part of the Alpine region. Particularly the DTMs of Piedmont, Lombardy and Switzerland were available. The paper has discussed the operations needed for the final merging. At first, all the DTMs have been transformed to the same 3D reference frame, that is the European ETRF2000. Then, several crosschecks have been performed: in particular, the LR DTMs have been compared in their overlapping boundaries. Local anomalies have been identified that, contrary to the expectations, are not distributed in the more rough areas but seem due to problems in the generation of the models. Moreover, a bias of about 1.5 meter exists between Piedmont and the other two DTMs: the bias is smaller than the nominal accuracy of all the datasets but it is everywhere present.

The first product of HELI-DEM is HD-1, a unified DTM obtained through the merging of the available low / medium resolution DTMs. HD-1 covers the ETRF2000 rectangle comprised by the following boundaries: $\lambda=7.80^{\circ}$ East and $\lambda ? 10.70^{\circ}$ East, $\phi=45.10^{\circ}$ North and $\phi=46.70^{\circ}$ North. It is gridded in geographical coordinates with a spatial resolution of $2 \times 10^{-4}$ degrees. It has been obtained by re-gridding all the input low resolution in the final grid and averaging the results in their overlap areas. One high resolution DTM was available for the alpine valleys and has been used to correct HD-1, at least locally where it exists. The correction has been performed studying a suitable method to avoid discontinuities at the borders. The resulting DTM is named HD-2 and has been externally validated by RTK GNSS measures: they confirm the good quality of the applied corrections.

At the present, HD-1 and HD-2 are being compared with the ASTER/SRTM/GMTED2010 global models. The results obtained by these comparisons will be presented at the symposium and discussed in a future paper.

\section{ACKNOWLEDGMENTS}

This research is a part of the HELI-DEM (Helvetia-Italy Digital Elevation Model) project, funded by the European Regional Development Fund (ERDF) within the Italy-Switzerland cooperation program. More details about the project can be found on the website: www.helidem.eu.

\section{References}

Altamimi, Z. and C. Boucher, The ITRS and ETRS89 Relationship: New Results from ITRF2000, Report on the Symposium of the IAG Subcommission for Europe (EUREF), Dubrovnik, 2001

Barzaghi R., Borghi A., Carrion D., Sona G. Refining the estimate of the Italian quasi-geoid. Bollettino di Geodesia e Scienze Affini, ISSN 0006-6710, fascicolo 3/2007, pp. 145-159, 2007

Benciolini B., Biagi L., Crespi M., Manzino A., Roggero M., Reference frames for GNSS positioning services: some problems and proposals. Journal of Applied Geodesy, N. 22008

Biagi L., Brovelli M.A., Campi A., Cannata M., Carcano L., Credali M., De Agostino M., Manzino A., Sansò F., Siletto G. Il progetto HELI-DEM (Helvetia-Italy Digital Elevation Model): scopi e stato di attuazione, in Bollettino della Società Italiana di 
Fotogrammetria e Topografia, $\mathrm{n}^{\circ} 1 / 2011$, pp. 35-51, ISSN: 1721-971X, 2011

Biagi L., Carcano L., De Agostino M.. DTM cross validation and merging: problems and solutions for a case study within the HELI-DEM project. International Conference ISPRS, Melbourne., 25 August-1 September 2012, 2012a

Biagi L., Carcano L., Lucchese A., Negretti M. Creation of a multiresolution and multiaccuracy DTM: problems and solutions for HELI-DEM case study. ISPRS Symposium: The Role of Geomatics in Hydrogeological Risk. Padua, 2013

Biagi L., Carcano L.. Regridding and merging overlapping DTMs: methodological problems and solutions in HELI-DEM. accepted for the publication in the VIII Hotine Marussi proceedings, IAG Symposia Volumes series. in press

Biagi L., Dabove P., Manzino A., Taglioretti C., Visconti M.G.. Il posizionamento NRTK in alta quota: applicazioni e risultati nell'ambito del progetto Helidem. Bollettino della società italiana di fotogrammetria e topografia , vol. 3/2012,p. 9-26, $2012 b$

Boucher C. and Altamimi Z.. Memo: Specifications for reference frame fixing in the analysis of a EUREF GPS campaign, http://etrs89.ensg.ign.fr/memo-V8.pdf, 2011

Brigham E.O. The Fast Fourier Transform. by Prentice-Hall, Inc. Englewood Cliffs, N. J., 1974

Brovelli M. A., Cannata M., and Longoni U., LIDAR data filtering and DTM interpolation within GRASS, Transactions in GIS, vol. 8, no. 2, pp. 155-174, 2004
Carcano L. Merging local DTMS: HELI-DEM project, problems and solutions. PhD Thesis. 2014

Donatelli D., Maseroli R., Pierozzi M., La trasformazione tra sistemi di riferimento utilizzati in Italia, Bollettino di Geodesia e Scienze Affini, Anno LXI, Nº 2, 2002

El-Sheimy N., Valeo C., Habib A.. Digital Terrain Modeling Acquisition, manipulation and applications. Artech House, 2005

Hofmann-Wellenhof D.B., Lichtenegger H., Wasle E. GNSS Global Navigation Satellite Systems: GPS, GLONASS, Galileo, and more. ISBN 978-3-211-73012-6 Springer Wien New York, 2008

Koch K. R., Parameter estimation and hypothesis testing in Linear models, Springer Verlag, 1987

Li Z., Zhu Q., and Gold C. Digital terrain modeling: principles and methodology. CRC, 2005

Marti, U. Comparison of High Precision Geoid Models in Switzerland. Dynamic Planet, International Association of Geodesy Symposia, 2007, Volume 130, Part III, pp. 377-382.

Rees W.G., The accuracy of digital elevation models interpolated to higher resolutions. International Journal of Remote Sensing, vol. 21, no.1, pp. 7-20, 2000

Surace L.. La nuova rete geodetica nazionale IGM95: risultati e prospettive di utilizzazione, Bollettino di Geodesia e Scienze affini, Anno LVI, N², 1997

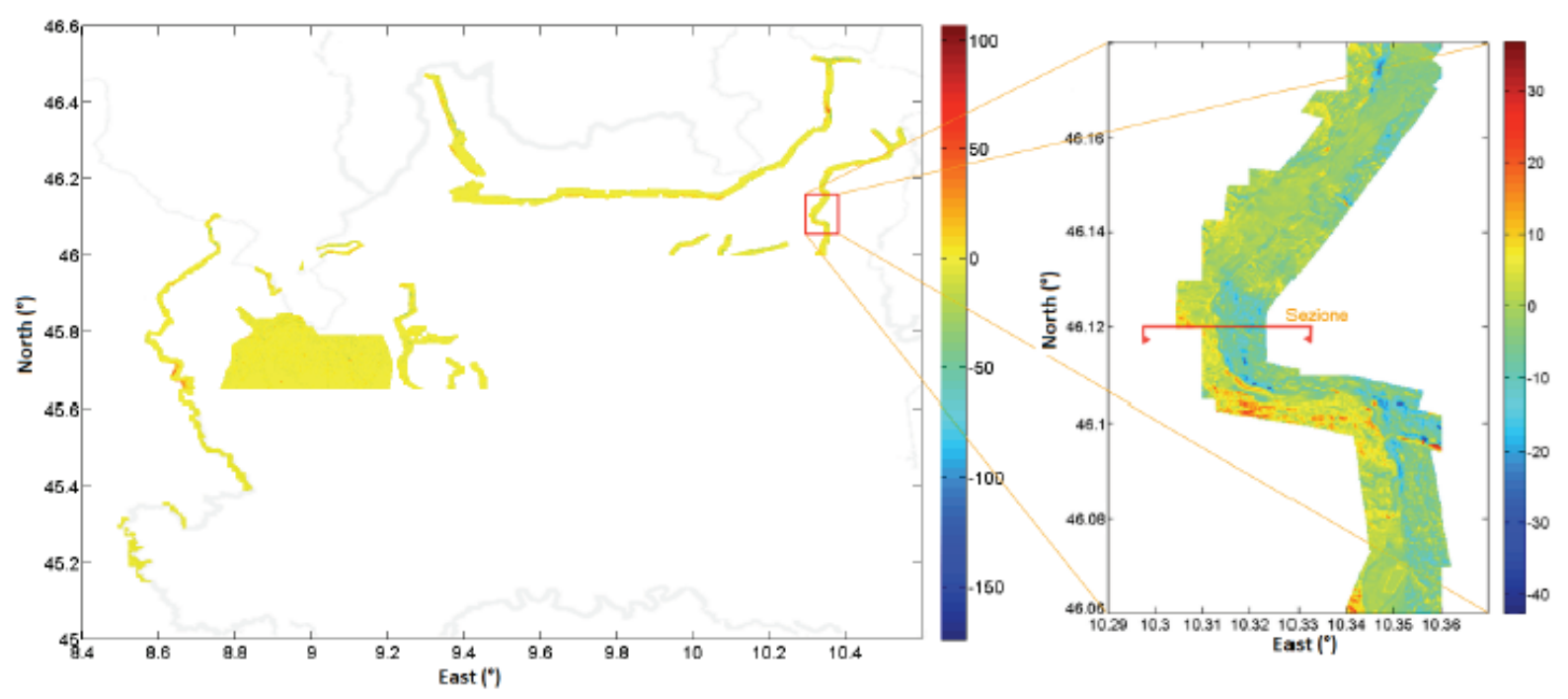

Figure 3. On the left: coverage of PST-A DTM and its differences with HD-1. At the right: a zoom of one example zone. Differences values in meters. 